\title{
Rydberg entangling gates in silicon
}

\author{
E. Crane $\odot,{ }^{1,{ }^{*}}$ A. Schuckert, ${ }^{2}$ N. H. Le,${ }^{3}$ and A. J. Fisher ${ }^{4}$ \\ ${ }^{1}$ Department of Electrical Engineering and London Centre for Nanotechnology, \\ University College London, Gower Street, London WC1E 6BT, United Kingdom \\ ${ }^{2}$ Department of Physics, Technical University of Munich, 85748 Garching, Germany \\ ${ }^{3}$ Advanced Technology Institute and Department of Physics, University of Surrey, Guildford GU2 7XH, United Kingdom \\ ${ }^{4}$ Department of Physics and Astronomy and London Centre for Nanotechnology, \\ University College London, Gower Street, London WC1E 6BT, United Kingdom
}

(Received 2 September 2020; accepted 14 May 2021; published 23 July 2021)

\begin{abstract}
In this paper we propose a Rydberg entangling gate scheme which we demonstrate theoretically to have an order-of-magnitude improvement in fidelities and speed over existing cold atom protocols. It requires a large Rabi frequency compared to the interaction strength, which is difficult in cold atoms, but natural in donors in silicon, where it could help overcome the strenuous requirements on atomic precision donor placement and substantial gate tuning, which so far has hampered scaling. Furthermore, the gate operation would be ultrafast, on the order of picoseconds. We calculate multivalley van der Waals, induced electric dipole and total Rydberg interactions for several donor species using the finite-element method and show that they are important even for low-lying excited states. We show that Rydberg gate operation is possible within the lifetime of donor excited states with $99.9 \%$ fidelity for the creation of a Bell state in the presence of decoherence.
\end{abstract}

DOI: 10.1103/PhysRevResearch.3.033086

\section{INTRODUCTION}

Donor electron spin qubits in silicon are competitive in most of Di Vincenzo's criteria for a quantum computer implementation: scalability (and complementary metal oxide semiconductor, CMOS, compatibility), high-fidelity initialization and readout, as well as extremely long coherence times [1-5]. However, published experimental results of entangling gates in silicon report only up to $86 \%$ [6] or 90\% [7] Bell-state preparation fidelity. Moreover, entangling exchange interactions can change by orders of magnitude if the distance between orbital ground-state spins is varied by one lattice site [8]. Due to the impact on gate durations, this renders scaling challenging, as even hydrogen lithography cannot to date perform donor placement accurately to within a single silicon lattice site $[9,10]$.

In this paper we draw on the analogy between donors trapped in the silicon lattice and alkali Rydberg atoms trapped in light fields [12] to propose a Rydberg entangling gate which is robust to variations in the interqubit distance, with theoretical fidelities up to $99.9 \%$ in phosphorus ( $\mathrm{Si}: \mathrm{P})$, arsenic (Si:As), and ionized selenium (Si:Se+) donors in silicon. Existing protocols make different assumptions about three key inverse timescales: the rate of damping $T_{1}^{-1}$, the frequency

\footnotetext{
*e.crane@ucl.ac.uk

Published by the American Physical Society under the terms of the Creative Commons Attribution 4.0 International license. Further distribution of this work must maintain attribution to the author(s) and the published article's title, journal citation, and DOI.
}

of driving $\Omega$, and the strength of the blockade interaction $u$. Our gate relies on the enhancement of the Rabi frequency $\Omega$ and of the interactions $u$ between atoms in an orbital excited (Rydberg) state $|r\rangle$, which leads to rapid gate operations on the order of hundreds of picoseconds. We show that the theoretical fidelities achievable by our gate protocol are an order of magnitude higher than existing Rydberg atom schemes. This is because our gate allows for the excited-state interaction $u$ to be on the same order of magnitude as the Rabi frequency $\Omega$ coupling one of the qubit levels $|1\rangle$ spin-selectively (see Fig. 1) to $|r\rangle$.

Specifically, we show here that while in cold atoms excitation to high-lying excited states is needed to induce strong dipole interactions, for donors in silicon the interactions generated by the low-lying excited states are large enough due to the large dielectric screening and small effective mass in silicon. We calculate electric (not magnetic [13]) dipole and van der Waals interactions between excited states. The finite-element method (FEM) enables quick and accurate calculations of large numbers of silicon donor wave functions of high-lying excited states (with or without an electric field) within the effective mass approximation, required for perturbation theory. To predict the fidelities we simulate the entangling gate operation in the presence of a finite excitedstate lifetime $T_{1}$, assuming the dephasing time to be twice the decay time, and show that our proposal outperforms existing Rydberg schemes. By using the experimentally determined $T_{1}$ and our numerically determined $u$, we find that high fidelities independent of placement errors can be reached in silicon. Lastly, we show that this gate is robust to variations in donor energy levels (inhomogeneous broadening) and that it only requires tuning of a single independent parameter. 
(a)

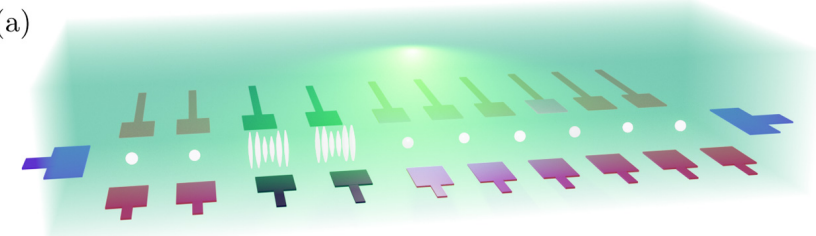

(b)

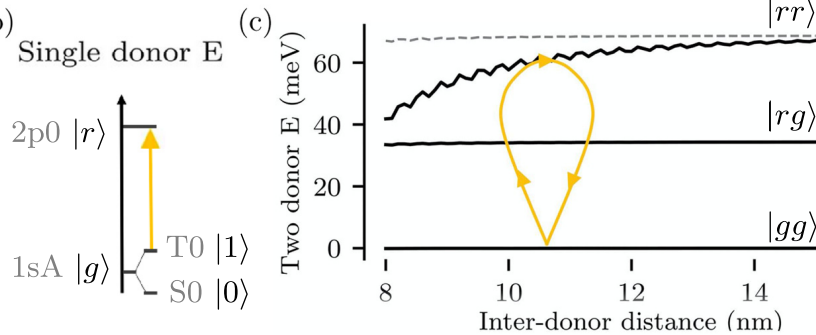

FIG. 1. Rydberg gate in silicon. (a) Illustration of the Rydberg entangling gate silicon quantum computer showing the global laser tuned to the $|g\rangle \rightarrow|r\rangle$ transition. The entangled qubit pair is selected by locally Stark shifting all other donors off resonance (red gates). Global gates (blue) enable use of induced dipolar interactions. Readout devices can be placed on the layer below the one shown here. This scheme can be extended for a surface code quantum computer similarly to [11]. (b) The ground-state hyperfine levels are used as a qubit basis, with a higher-lying orbital excited state as $|r\rangle$. (c) The interactions between two atoms in the excited state barely oscillate, leading to a distance-robust, two-qubit phase acquired after a Rabi cycle (residual oscillations are due to multivalley interference in the exchange interaction).

\section{Rydberg entangling gates}

Whereas previous gate schemes [14-16] (presented in Appendix A) required $\hbar / T_{1} \ll \Omega<u$, our proposal only requires $\hbar / T_{1} \ll \Omega, u$, allowing for much smaller gate durations and hence less probability of decay from $|r\rangle$, leading to higher gate fidelities. This is because previous schemes were based on the blockade, in which the doubly excited state $|r r\rangle$ is tuned out of resonance due to strong interactions induced by $\Omega \ll u$. Therefore the gate duration $\tau \sim 1 / \Omega$ is limited to $\tau \gg 1 / u$. In our protocol, due to $\Omega$ being on the same order of magnitude as $u$, the gate duration can be $\tau \sim 1 / u$. We note that in cold atom platforms $\Omega \ll u$ is always given, such that our gate most likely does not yield substantial advantage. However, in the donor platform $u$ is the limiting parameter, leading to a substantial improvement.

The pulse sequence, represented in Fig. 2, consists of acting twice with a pulse with Rabi frequency $|\Omega|$ detuned from the $|r\rangle$ transition on both donors simultaneously for a duration chosen such that the first pulse returns $|01\rangle$ and $|10\rangle$ to themselves. The phase $\xi$ of $\Omega$ is then chosen such that $|11\rangle$ returns to itself after a second pulse of the same duration and Rabi frequency $|\Omega|$. During the two pulses, $|11\rangle$ picks up a two-qubit phase due to the interactions in $|r r\rangle$. Lastly, the detuning $\Delta$ is chosen such that the final phases of all two-qubit states implement a controlled phase gate. Global addressing enables parallel entangling gate operations as demonstrated in Ref. [16]. Conditions for a successful gate implementation are spin-selective excitation to $|r\rangle$ (either leaving the $|0\rangle-|r\rangle$

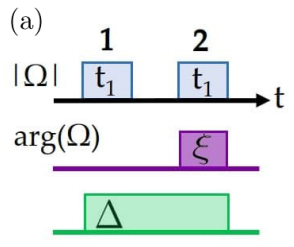

$$
\text { (b) } \begin{aligned}
|00\rangle & \rightarrow|00\rangle \\
|01\rangle & \rightarrow|01\rangle e^{i \phi} \\
|10\rangle & \rightarrow|10\rangle e^{i \phi} \\
|11\rangle & \rightarrow|11\rangle e^{i(2 \phi-\pi)}
\end{aligned}
$$

FIG. 2. Pulse sequence. (a) Global pulse sequence acting with $|\Omega|$ on both qubits simultaneously: pulse duration $\tau$ chosen such that the first pulse returns $|01\rangle$ to itself (whereas $|11\rangle$ is left in an arbitrary location on the Bloch sphere), $\xi$ is the phase of the second pulse chosen such that $|11\rangle$ returns to itself after the second pulse, $\Delta$ detuning of the $|1\rangle$ to $|r\rangle$ transition chosen such that the phases highlighted in (b) are equal.

transition off resonance or forbidden by a selection rule) and $u, \Omega>\hbar / T_{1}$. In the following, we show that these two conditions can be fulfilled in both shallow and deep donors.

\section{REALIZING A RYDBERG GATE IN SILICON}

\section{A. Spin-selective excitation to $|r\rangle$}

The singly ionized deep double donor selenium $\left({ }^{77} \mathrm{Se}^{+}\right)$has a large hyperfine interaction in the ground state, giving rise to singlet $\mathrm{S} 0$ and triplet $\mathrm{T} 0$ states as the qubit basis with $T_{1}=4.6$ hours at around $2 \mathrm{~K}$ [17] and a lower bound on $T_{2}$ of $2 \mathrm{~s}$ at around $4 \mathrm{~K}$ [5]. For $|r\rangle$ both the \pm valley states of $1 s T_{2} \Gamma_{7}$ with $T_{1}=7.7 \mathrm{~ns}$ around $4 \mathrm{~K}$ [17] and $2 p 0$ can be used, as they are dipole-allowed spin-selective transitions with easily accessible excitation energies.

For the shallow donors Si:P, Si:As, antimony, and bismuth, the qubit can be encoded in the hyperfine S0 and T0 states with a T2 time exceeding $7 \mathrm{~s}$ at around $2 \mathrm{~K}$ and a splitting of around $117 \mathrm{MHz}$ in zero field [3]. For $|r\rangle$ we take the $2 p 0$ state with a $T_{1}=0.235 \mathrm{~ns}$ up to $5 \mathrm{~K}$ [22], which can be selectively addressed from the hyperfine ground states due to the negligible hyperfine splitting in the $2 p 0$ state. An alternative could be using a two-photon excitation via the D0X level $[3,19]$ or using micromagnets to emulate spin-orbit coupling for spin-selective excitation to the $2 p 0$ state, as the larger extent of the $2 p 0$ wave function would lead to a larger Zeeman splitting in $|r\rangle$ than in $|g\rangle$ [24]. Higher-lying states would give rise to larger $u$, at the cost of a smaller $\Omega$ or the need for a two-photon excitation, as is the case for cold atoms. For all proposals we have checked that $\Omega>\hbar / T_{1}$ is feasible with laser intensities below $1 \mathrm{~W} / \mu \mathrm{m}^{2}$, and we list their parameters in Table I, and having discussed that spin-selective excitation of orbital excited states is indeed possible, we now calculate the interactions between donors in $|r\rangle$ to find $u$.

\section{B. Rydberg interaction $u$}

Entangling gate schemes in donors so far have focused on harnessing the highly oscillatory and exponentially decaying ground-state ferromagnetic exchange $(J)[6,25]$ : this interaction is negligible in high-lying excited states of Rydberg atoms, where $u$ is dominated by the electric dipole interaction 
TABLE I. Properties of orbital excited states used in this work. (a) Calculated using FEM results. (b) From [17]. (c) From [18]. Two-photon excitation via the D0X state could also be envisaged $\left(d_{|1\rangle \rightarrow|D 0 X\rangle}=0.04\right.$ Debye and $d_{|D 0 X\rangle \rightarrow|r\rangle}=0.003$ Debye [19]). (d) We assume a conservative $T_{1}=1 \mathrm{~ns}$. (e) From [20]. (f) From [17]. Photoluminescence radiative $T_{1}=900 \mathrm{~ns}$; however, modulationfrequency-dependent luminescence direct $T_{1}=7.7 \mathrm{~ns}$ [17] (may be limited by thermal phonons leaving room for improvements in $T_{1}$ [21]). (g) From [22]. Dominated by phonon-assisted relaxation. (h) From [17]. Limited by $T_{1}$. (j) From [23]. The sample was not isotopically pure, leaving room for improvements in $T_{2}$.

\begin{tabular}{lccc}
\hline \hline $\begin{array}{l}\text { Donor } \\
|r\rangle\end{array}$ & $\mathrm{Se}+$ & $\mathrm{Se}+$ & $\mathrm{P} / \mathrm{As}$ \\
$2 p 0$ \\
\hline$d_{|1\rangle \rightarrow|r\rangle}($ Debye $)$ & $2 p 0$ & $1 s T 2$ & $31^{\mathrm{c}}$ \\
$\hbar \omega_{|1\rangle \rightarrow|r\rangle}(\mathrm{meV})$ & $0.97^{\mathrm{a}}$ & $1.96^{\mathrm{b}}$ & 34 \\
Theo. $T_{1}(\mathrm{~ns})$ & 548 & 427 & $1.1 / 1.8^{\mathrm{e}}$ \\
Exp. $T_{1}(\mathrm{~ns})$ & $-^{\mathrm{d}}$ & - & $0.235^{\mathrm{g}}$ \\
Exp. $T_{2}(\mathrm{~ns})$ & $-^{\mathrm{d}}$ & $7.7^{\mathrm{f}}$ & $0.160^{\mathrm{j}}$ \\
\hline \hline
\end{tabular}

$$
V_{d}=\frac{\boldsymbol{p}_{1} \boldsymbol{p}_{2}-3\left(\boldsymbol{n} \cdot \boldsymbol{p}_{1}\right)\left(\boldsymbol{n} \cdot \boldsymbol{p}_{2}\right)}{R^{3}},
$$

where $\boldsymbol{p}_{\boldsymbol{i}}$ is the dipole operator of atom $i, R$ is the interatomic distance, and $\boldsymbol{n}$ is the unit vector pointing along the interdonor axis. In the absence of an electric field, neutral atoms and donors do not have a permanent dipole moment so $V_{d}$ only contributes at second order in perturbation theory: van der Waals $\left(V_{\mathrm{VdW}}\right)$ interactions falling off as $\sim 1 / R^{6}$ whose strength is determined by the polarizability which scales with the principal quantum number as $n^{11}$. Contrarily, $J$ is determined by the size of the wave functions which scale as $n^{2}$ and decay exponentially with interdonor distance. Therefore dipolar interactions dominate for large principal quantum number and distance.

In more detail, in this work we consider the tight-binding model, with additional dipolar forces due to a donor being made up of a core plus a valence electron. The tight-binding model is written

$$
H=\sum_{i \neq j} a_{i \sigma}^{\dagger} t_{i j} a_{j \sigma}+\sum_{i i^{\prime} j j^{\prime}} \frac{U_{i i^{\prime} j j^{\prime}}}{2} a_{i \sigma}^{\dagger} a_{i^{\prime} \sigma^{\prime}}^{\dagger} a_{j^{\prime} \sigma^{\prime}} a_{j \sigma},
$$

where $t$ is the single hopping matrix element between neighboring sites and

$$
\begin{aligned}
U_{i i^{\prime} j j^{\prime}}= & \int d^{d} \boldsymbol{r}_{1} d^{d} \boldsymbol{r}_{2} \psi_{R_{i}}^{*}\left(\boldsymbol{r}_{1}\right) \psi_{R_{i}^{\prime}}^{*}\left(\boldsymbol{r}_{2}\right) V\left(\boldsymbol{r}_{1}-\boldsymbol{r}_{2}\right) \\
& \times \psi_{R_{j}}\left(\boldsymbol{r}_{1}\right) \psi_{R_{j}^{\prime}}\left(\boldsymbol{r}_{2}\right),
\end{aligned}
$$

where $V$ is the Coulomb interaction, given by $V(\boldsymbol{r})=V_{0} / \boldsymbol{r}$, where $V_{0}=\frac{e^{2}}{4 \pi \epsilon_{0} \epsilon_{S}}, e$ is the electron charge, and $\epsilon_{S}=11.4$ is the dielectric constant of silicon.

\section{Intersite Coulomb electron-electron repulsion}

The contribution from $U_{i i^{\prime} i i^{\prime}}=W_{i i^{\prime}}$ is $\sum_{i \neq i^{\prime}} W_{i i^{\prime}} \hat{n}_{i} \hat{n_{i^{\prime}}}$, where $\hat{n}_{i}=\sum_{\sigma} a_{i \sigma}^{\dagger} a_{i \sigma}$, which corresponds to the essentially classical Coulomb interactions between donors on neighboring sites.
From this we get the intersite Coulomb electron-electron repulsion:

$$
W_{12}=V_{0} \iint d \boldsymbol{r}_{1} d \boldsymbol{r}_{2} \frac{\left|\psi_{1}\left(\boldsymbol{r}_{1}\right)\right|^{2}\left|\psi_{2}\left(\boldsymbol{r}_{2}-\boldsymbol{R}\right)\right|^{2}}{\left|\boldsymbol{r}_{1}-\boldsymbol{r}_{2}\right|},
$$

where $\boldsymbol{R}=\boldsymbol{R}_{2}-\boldsymbol{R}_{1}$ is the separation vector between the two donors. The contribution from $U_{i i i i}$ corresponds to the on-site interaction, which can lead to antiferromagnetic superexchange which is negligible in our case. We do not include here electron-core interactions [26,27], as they will cancel in the expression of the total interaction.

\section{Ferromagnetic exchange coupling}

The contributions from $U_{i j j i}$ can be rewritten, making use of Pauli matrix identities, into

$$
\sum_{i \neq j} \frac{U_{i j j i}}{2} a_{i \sigma}^{\dagger} a_{j \sigma^{\prime}}^{\dagger} a_{i \sigma^{\prime}} a_{j \sigma}=-\sum_{i \neq j} U_{i j j i}\left(\overrightarrow{S_{i}} \cdot \overrightarrow{S_{j}}+\frac{1}{4} \hat{n}_{i} \hat{n}_{j}\right) \text {. }
$$

It corresponds to the ferromagnetic exchange coupling. Note that for calculating the total interaction for the gate, we are only interested in the case where both spins are on resonance with the laser, so both spins are aligned. This leads to $\overrightarrow{S_{i}}$. $\overrightarrow{S_{j}}=\frac{1}{4} \hat{n}_{i} \hat{n}_{j}$. We refer to $U_{i j j i}$ as $J_{i j}$ and we have

$$
\begin{aligned}
J_{12}= & \int d \boldsymbol{r}_{1} d \boldsymbol{r}_{2} \psi_{1}^{*}\left(\boldsymbol{r}_{1}\right) \psi_{2}^{*}\left(\boldsymbol{r}_{2}-\boldsymbol{R}\right) \frac{V_{0}}{\left|\boldsymbol{r}_{1}-\boldsymbol{r}_{2}\right|} \\
& \times \psi_{2}\left(\boldsymbol{r}_{1}-\boldsymbol{R}\right) \psi_{1}\left(\boldsymbol{r}_{2}\right),
\end{aligned}
$$

where $\boldsymbol{R}=\boldsymbol{R}_{2}-\boldsymbol{R}_{1}$ is the separation vector between the two donors. The leading correction to this electron-only model of donor interactions is given by dipole interactions.

\section{Dipole-dipole interactions}

Dipole moments of a state can be calculated as the derivative of the Stark shift of that state (which we study in more detail in Appendix D 2). Because donors do not have permanent dipole moments, they require electric fields in order to have dipole-dipole interactions, which take the form

$$
V_{d d}=-\sum_{n^{\prime}, l^{\prime}, j^{\prime}, m^{\prime}} \frac{\left\langle n^{\prime}, l^{\prime}, j^{\prime}, m^{\prime}\left|V_{d}\right| n, l, j, m\right\rangle}{E_{\left|n^{\prime}, l^{\prime}, j^{\prime}, m^{\prime}\right\rangle}-E_{|n, l, j, m\rangle}},
$$

where $\boldsymbol{p}_{\boldsymbol{i}}$ is the dipole moment of atom $i, R$ is the interatomic distance, and $\boldsymbol{n}$ is the unit vector between the two donors.

\section{Van der Waals}

A dipole can furthermore be induced by the vacuum, leading to van der Waals forces, which are calculated in second-order perturbation theory of $V_{d}$, forces

$$
\begin{aligned}
& V_{V d W i j}=-\sum_{\substack{n^{\prime}, l^{\prime}, j^{\prime}, m^{\prime} \\
n^{\prime \prime}, l^{\prime \prime}, j^{\prime \prime}, m}} \\
& \frac{\left|\left\langle n^{\prime}, l^{\prime}, j^{\prime}, m^{\prime}\left|\otimes\left\langle n^{\prime \prime}, l^{\prime \prime}, j^{\prime \prime}, m^{\prime \prime}\left|V_{d}\right| n, l, j, m\right\rangle \otimes\right| n, l, j, m\right\rangle\right|^{2}}{E_{\left|n^{\prime}, l^{\prime}, j^{\prime}, m^{\prime}\right\rangle}+E_{\left|n^{\prime \prime}, l^{\prime \prime}, j^{\prime \prime}, m^{\prime \prime}\right\rangle}-2 E_{|n, l, j, m\rangle}} .
\end{aligned}
$$


The Hamiltonian we use in this problem therefore corresponds to

$$
H=\sum_{i \neq j} \frac{1}{2}\left(W_{i j}+V_{V d W i j}-J_{i j}\right) \hat{n}_{i} \hat{n}_{j}
$$

In the presence of an electric field, $W$ is dropped in favor of $V_{V d d}$, which becomes the leading contribution.

\section{Donor wave functions}

In silicon, the six conduction-band minima lead to donor electron multivalley wave functions. Here, we calculate multivalley results for $J$, electron-electron repulsion $(W)$, and also $V_{V d W}$ and the induced dipole interaction $V_{d d}$ in the presence of an electric field. We employ the FEM to solve the Schrödinger equation exactly within effective mass theory:

$$
\left[-\frac{\hbar^{2}}{2 m^{*}} \nabla^{2}+U_{\mathrm{imp}}(r)+U_{c c}(r)+U_{E f}(r)\right] \psi_{i}(r)=\epsilon_{i} \psi_{i}(r),
$$

where $U_{c c}$ is a central-cell potential for the $S$ states, which have a non-negligible probability of being at the core, $U_{E f}$ is the electric field, $\epsilon_{i}$ is the binding energy of the donor, and $m^{*}$ is the effective mass of the electron in the silicon lattice. Then we couple the result into the six valleys. The FEM solves for any number of eigenstates, enabling a convergence check of perturbative calculations. For $V_{V d W}$ interactions, we typically include $\sim 40$ states. Similarly, we obtain $V_{d d}$ from the dipole moment, which we get from the slope of the multivalley Stark shift. This perturbatively calculated Stark shift does not contain contributions from the continuum, which we quantify by comparing to the single-valley energies directly generated by the FEM while varying $U_{E f}$ (see Appendix B). As the outermost donor electron is very weakly bound to the core, we set the maximally applicable electric field (which wouldn't ionize $|r\rangle$ during its lifetime) by generalizing the method in Ref. [28] to donors in silicon, see Appendix D 1, consistent with experimental results.

\section{Rydberg interaction $u$ results in donors}

In the case of the entangling gate, we are interested in total Rydberg interaction $u$, which corresponds to the energy difference between both donors being excited to the Rydberg state and none or only a single of the two being excited. We use the notation $E_{|r r\rangle}$ for both spins being excited, $E_{|r g\rangle}$ for one donor being excited, and the other remaining in the ground state and $E_{|g g\rangle}$ for both donors in the ground state:

$$
\begin{gathered}
E_{|r r\rangle}=W_{|r r\rangle}+V_{V d W|r r\rangle}-J_{|r r\rangle}, \\
E_{|r g\rangle}=W_{|r g\rangle}, \\
E_{|g g\rangle}=W_{|g g\rangle},
\end{gathered}
$$

where, again, $V_{V d d}$ dominates $W$ in the presence of an external electric field, and we neglected the extremely small contributions coming from $V_{V d W}$ and $J$ for $|r g\rangle$ and $|g g\rangle$. From these expressions we are able to obtain the expression for $u$, the additional energy experienced by the atoms when they are
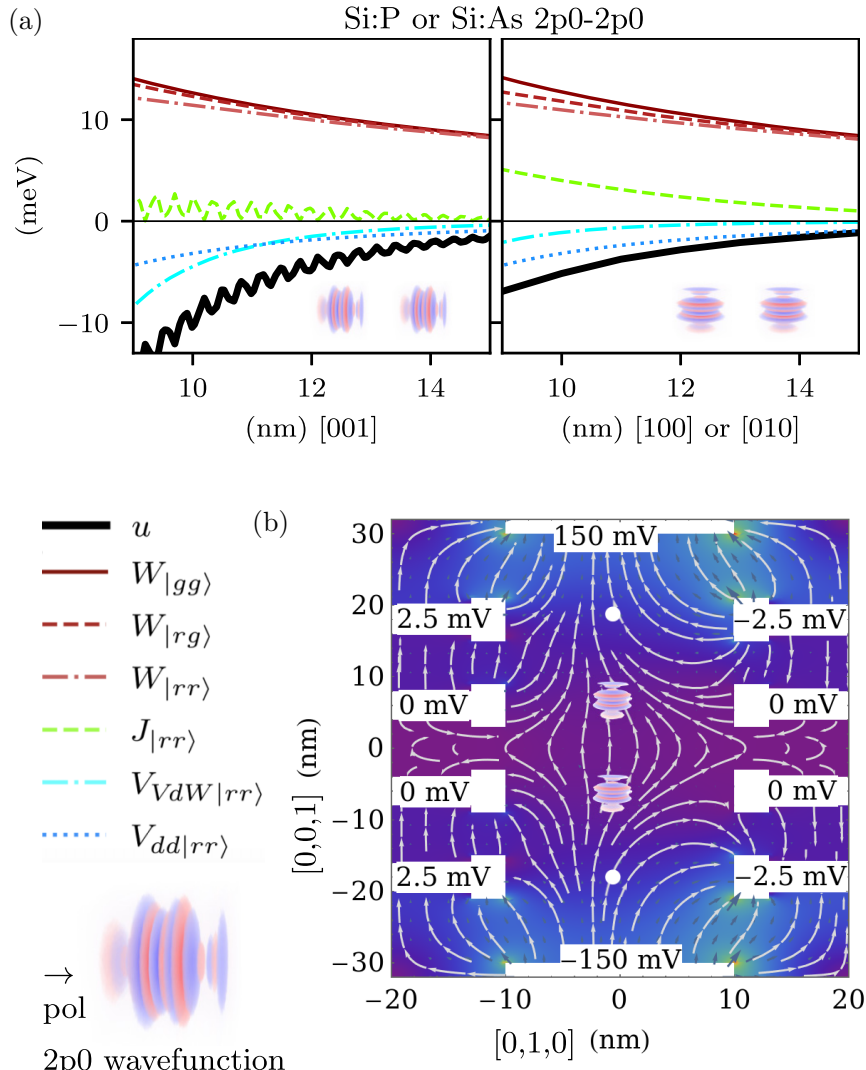

$2 \mathrm{p} 0$ wavefunction

FIG. 3. Rydberg interaction energy $(u)$ and electric fields. (a) Interactions in $\mathrm{meV}$ between As or $\mathrm{P}$ donors in this same setup. $u$ : total Rydberg interaction, $W$ : electron-electron repulsion, $J$ : ferromagnetic exchange, $V_{V d W}$ : van der Waals, $V_{d d}$ : induced dipole interaction at $0.18 \mathrm{~V} / \mu \mathrm{m}$, see Appendix D 2. "r" and "g" refer to Rydberg and ground state as defined in Fig. 1. $2 p 0$ wave-function illustration (blue: negative; red: positive), showing multivalley oscillations along the polarization direction only. (b) Electrostatic simulation of a device (assuming a 2D layer with insulators above and below). Top and bottom global gates apply $0.18 \mathrm{~V} / \mu \mathrm{m}$ (very low ionization probability, see Appendix D 1) between the two central donors, inducing dipolar interactions. The top and bottom donors are shifted off resonance with the laser due to side gates applying a field of $0.5 \mathrm{~V} / \mu \mathrm{m}$ (as in Fig. 1). Electric field from 0 to $3 \mathrm{~V} / \mu \mathrm{m}$. Polarization parallel to interdonor axis $\{0,0,1\}$ in all figures. For more information, see Appendix D 3 .

doubly excited as opposed to singly excited:

$$
\begin{aligned}
u & =\left(E_{|r r\rangle}-E_{|g r\rangle}\right)-\left(E_{|g r\rangle}-E_{|g g\rangle}\right) \\
& \approx W_{|r r\rangle}-2 W_{|r g\rangle}+W_{|g g\rangle}-J_{|r r\rangle}+V_{V d W|r r\rangle},
\end{aligned}
$$

$J_{|g g\rangle}, V_{V d W|g g\rangle}, V_{V d W|r g\rangle}, J_{|r g\rangle}$ are negligible for the range of donor separations considered in this work.

Figure 3 shows all interactions between two $2 p 0$ state Si:P or Si:As donors. The interaction energy is plotted as a map for the $2 p 0$ and $1 s T_{2} \Gamma_{7}$ states in $\mathrm{Si}: \mathrm{Se}+$ in Appendix $\mathrm{C}$ in Fig. 9. Contrary to the expectation from the ground orbital state and despite the small principal quantum number, both $V_{V d W}$ and $V_{d d}$ interactions are non-negligible. When two donors are aligned along the polarization and electric field axis as is shown in Fig. 3(a), $V_{V d W}$ and $V_{d d}$ dominate, with an 
oscillatory contribution from $J$. When aligned along an axis perpendicular to the polarization, $J$ and $V_{V d W}$ dominate, with no oscillations due to the $2 p 0$ state not showing multivalley oscillations in the plane perpendicular to the polarization axis.

We note that the contributions from $J$ are very small. This means that donors residing on different sublattices (which heavily affects $J$ but has only a small influence on other interaction types) has only a very small effect on $u$, as will be seen in Fig. 5 in the following section. For the $1 s T_{2} \Gamma_{7}$ state in $\mathrm{Si}: \mathrm{Se}+$, however, the dipole interactions are less dominant and $u$ is mainly given by $J$, as we show in Appendix C.

\section{E. Full device operation}

For a successful gate operation in a quantum computer, the interactions $u$ need to be switchable without affecting the surrounding qubits. Selecting the qubit pair to be excited to $|r\rangle$ requires Stark shifting the donors into resonance with the laser using electric fields [6,29], analogously to proposals for Rydberg atoms in optical lattices [30]. Single-qubit operations can be done in the same way using global microwave illumination [29]. In Fig. 3(b) we show our electrostatic simulation of the gate configuration sketched in Fig. 1(a). We found that the electric gate configuration sufficient for shifting donors off resonance would not create stray electric fields harmful to device operation, i.e., would not ionize the donors (see Fig. 10 in Appendix D).

In all donors and $|r\rangle$ studied here, $u$ is on the order of 1$10 \mathrm{meV}$, fulfilling $u>\hbar / T_{1}$. Given a large enough laser power, the interaction scale sets the ultimate limit to gate operation times, enabling entangling gate durations of a fraction of a nanosecond, nine to ten orders of magnitude faster than the qubit lifetime.

\section{FIDELITY OF BELL-STATE CREATION IN PRESENCE OF DECOHERENCE}

In order to show that high-fidelity entangling gates are possible in silicon within the short donor lifetimes (summarized in Table I), we calculate the fidelity of Bell-state creation $\mathcal{F}=\left\langle\Phi^{+}|\rho| \Phi^{+}\right\rangle$for various donors in the presence of decoherence by entering the interactions $u$ calculated in the previous section. To do this we model a two-donor system with the Hamiltonian [15]

$$
\begin{aligned}
H= & \sum_{i=1,2}\left(\frac{\Omega_{i}}{2}|1\rangle_{i}\left\langle r\left|+\frac{\Omega_{i}^{*}}{2}\right| r\right\rangle_{i}\langle 1|-\Delta| r\rangle_{i}\langle r|\right) \\
& +u|r\rangle_{1}\langle r|\otimes| r\rangle_{2}\langle r|,
\end{aligned}
$$

and simulate the full pulse sequence for creating the Bell state $\left|\Phi^{+}\right\rangle=\frac{1}{\sqrt{2}}|00\rangle+|11\rangle$ within a Markovian Lindblad master equation given by

$$
\partial_{t} \rho=-\frac{i}{\hbar}\left[H_{\mathrm{eff}} \rho-\rho H_{\mathrm{eff}}^{\dagger}\right]+\sum_{j} L_{j} \rho L_{j}^{\dagger},
$$

where $\rho$ is the two-donor density matrix (with each donor restricted to the states $|0\rangle,|1\rangle$, and $|r\rangle), L_{j}$ are the jump operators describing decoherence and $H_{\text {eff }}=H-\frac{i}{2} \sum_{j} L_{j}^{\dagger} L_{j}$. We include dephasing between $|1\rangle$ and $|r\rangle$ with rate $\gamma_{\mathrm{de}}=1 /\left(2 T_{1}\right)$ and spontaneous emission from the Rydberg state, with rate $\gamma_{\mathrm{se}}=1 / T_{1}$ :

$$
\begin{gathered}
L_{\mathrm{de}, 1}=\sqrt{\gamma_{\mathrm{de}}}[(|r\rangle\langle r|-| 1\rangle\langle 1|) \otimes \mathbb{1}], \\
L_{\mathrm{de}, 2}=\sqrt{\gamma_{\mathrm{de}}}[\mathbb{1} \otimes(|r\rangle\langle r|-| 1\rangle\langle 1|)], \\
L_{\mathrm{se}, 1}=\sqrt{\gamma_{\mathrm{se}}}[|1\rangle\langle r| \otimes \mathbb{1}], \\
L_{\mathrm{se}, 2}=\sqrt{\gamma_{\mathrm{se}}}[\mathbb{1} \otimes|1\rangle\langle r|],
\end{gathered}
$$

where we assumed spontaneous emission does not bring $|r\rangle$ to $|0\rangle$, and we neglect decoherence processes between the qubit levels, as these are negligible on the timescale of a single twoqubit gate.

We solve the evolution for arbitrary decoherence rates and interactions and deduce the necessary interaction strength and Rabi frequency for a successful gate operation. We start in the initial state $\left|\Psi_{0}\right\rangle=(|1\rangle+|0\rangle) / \sqrt{2} \otimes(|1\rangle+|0\rangle) / \sqrt{2}$ and entangle the two qubits by numerically solving the time evolution of the pulse sequence. Finally, we apply a single-qubit phase gate to rotate to $\left|\Phi^{+}\right\rangle[16]$. We assume the latter to be perfect, i.e., we act with the appropriate unitary operator on the density matrix instead of simulating the pulse. We use the fidelity of creating $\left|\Phi^{+}\right\rangle$, defined as $\mathcal{F}=\left\langle\Phi^{+}|\rho| \Phi^{+}\right\rangle$as a measure of how much decoherence alters the success of the gate.

In this paper we propose a "phase accumulation" variant of the "gate A" discussed in Jaksch et al. [15] and presented in Appendix A. To do so, we use the same pulse sequence as in the off-resonant blockade gate discussed in Levine et al. [16] and presented in Appendix A but reverse the reasoning-instead of tuning the gate time to the blockaded Rabi frequency, we use the unblockaded one, given by $\tau=2 \pi / \sqrt{\Omega^{2}+\Delta^{2}}$. Hence, the states $|01\rangle$ and $|10\rangle$ return to themselves after a single Rabi pulse, as can be seen in Fig. 4. Accordingly, we tune the phase $\xi$ such that the state $|11\rangle$ returns to itself after the second pulse, accumulating a two-qubit phase due to the nonvanishing probability to populate $|r r\rangle$, see Fig. 4.

That this proposal is advantageous compared to the blockade gates may seem counterintuitive at first due to the high loss rate from occupying $|r\rangle$, as opposed to avoiding it with the blockaded regime. However, in this gate the condition $\Omega<u$ is relaxed and hence the gate duration and hence the duration of occupation of $|r\rangle$ can be much smaller compared to the blockade gates, therefore reducing again the loss from $|r\rangle$ and increasing the fidelity.

We optimize the pulse sequence and find that the optimal Rabi frequency is proportional to the interaction strength. Furthermore, all other parameters are almost independent of the interaction strength. We obtain these results numerically by fixing $\tau=2 \pi / \sqrt{\Omega^{2}+\Delta^{2}}$ and optimizing $\Omega, \Delta$, $\xi$ for a given $u / \gamma$, obtaining the optimal pulse parameters shown in Fig. 4. In the large interaction limit, we find

$$
\begin{gathered}
\Omega / u=1.45747, \\
\Delta / \Omega=0.28757, \\
\xi=1.5306 .
\end{gathered}
$$


(a)

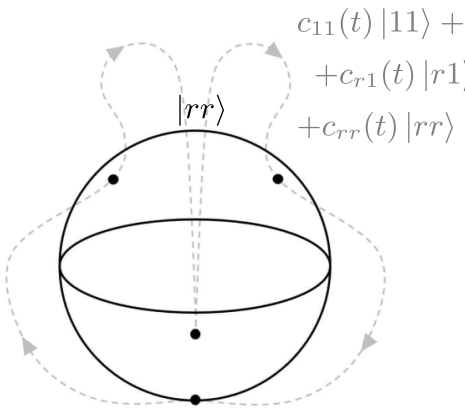

$|11\rangle$

(b)

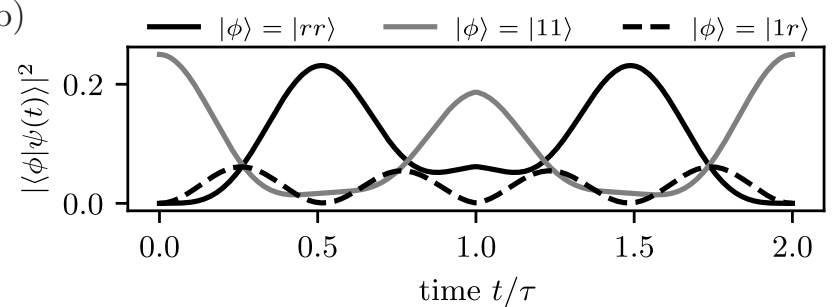

(c)

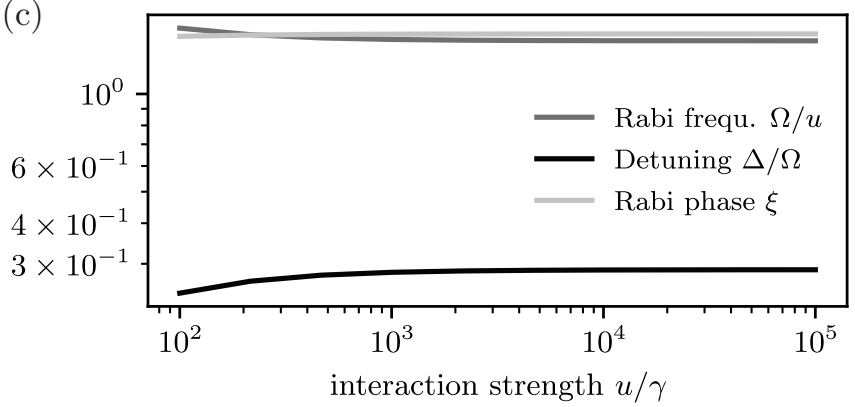

FIG. 4. Pulse sequence of proposed gate. (a) Bloch-sphere depiction of the dynamics of the states $|11\rangle$ and $|01\rangle$. The $|11\rangle$ state touches the Bloch sphere spanned by $|11\rangle$ and $|r r\rangle$ at times $(n / 2) \tau$ with $n=1,2,3,4$. This is more clearly shown in (b), which depicts the state probabilities when starting in the initial state $|\psi(0)\rangle=$ $(|0\rangle+|1\rangle) \otimes(|0\rangle+|1\rangle) / 2$. (c) Optimized pulse parameters in the blockade-inspired off-resonant gate. Note the very weak dependence on the interaction strength.

\section{Fidelities in the donor platform}

In Fig. 5, we analyse the Bell-state creation fidelity for our proposal and its dependence on the sublattice, the Rabi frequency, and the inhomogeneous broadening. In Fig. 6, we compare the Bell-state creation fidelity as a function of the distance between donors for our proposal and for the previous schemes proposed in the context of the cold atom platform. Importantly, fidelities of approximately $99.9 \%$ can be reached. Moreover, the fidelity is only weakly dependent on distance such that placement errors as large as several nanometers make little difference. As can be seen on Fig. 5 and Fig. 6, the following are all well within the width of the "fidelity resonance":

(i) Having both donors residing on different sublattices (which introduces large oscillations in the ferromagnetic exchange for schemes using ground-state interactions).

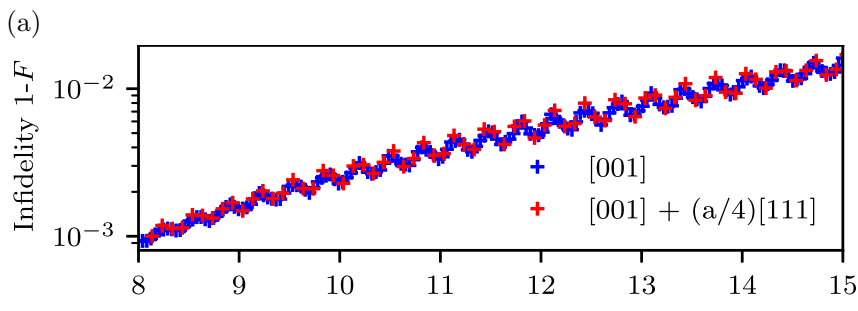

(b) (nm) $\{0,0,1\}$

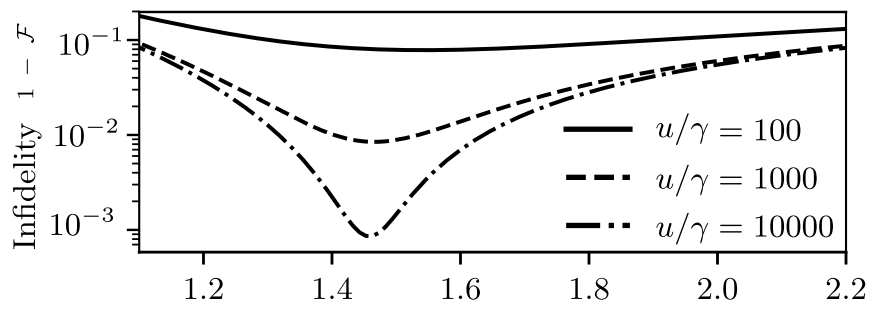

(c)

Rabi frequency $\Omega / u$

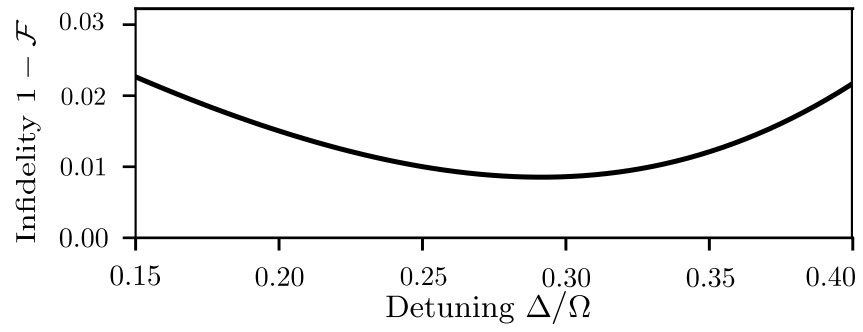

FIG. 5. Dependence of the fidelity on the sublattice, the Rabi frequency, and inhomogeneous broadening. (a) In the case of Si:P with $|r\rangle$ chosen as $2 p 0$, as is the case in the main text, the fidelity is robust to donors belonging to different silicon sublattices. Donors residing on different sublattices only heavily affects the exchange interaction. Because it is such a small contribution to the final blockade energy, the different sublattices barely affect the fidelity results. (b) Dependence of the fidelity on the Rabi frequency for the blockade-inspired off-resonant gate for some examples of the interaction strength. (c) Infidelity as a function of interaction strength scaled by $|r\rangle$ lifetime, for a fixed $\Omega$ and interaction strength $u$. Even a relatively large inhomogeneous broadening of $0.1 \Omega$ corresponding to roughly $0.2 \mathrm{meV}$ for $\mathrm{Si}: \mathrm{P}$, which is a factor of 2 larger than found in experiment [23], has very little effect on the fidelity.

(ii) A displacement within the $2 \times 3$ lattice site physical limit on the precision placement of hydrogen lithography of $\mathrm{Si}: \mathrm{P}[9]$,

(iii) A small diffusion out of plane (usually of around $2 \mathrm{~nm}$ for Si:P and $1 \mathrm{~nm}$ for Si:As [10]),

This result implies that a sample made with hydrogen lithography would not need tuning, assuming the interaction between donors as a function of distance is known, and for an ion-implanted sample, a rough scan of the sample with a scanning tunneling microscope (STM) after fabrication to determine the locations of the donors would suffice for finding good pulse parameters. Note that while placement insensitivity is stronger in the blockade gates, there is still an error introduced by $\Omega / u$, differing from the optimal value if $u$ varies.

Inhomogeneous broadening refers to a change in the excited-state energies due to a differing electrostatic environment of the donor, e.g., caused by randomly distributed silicon 

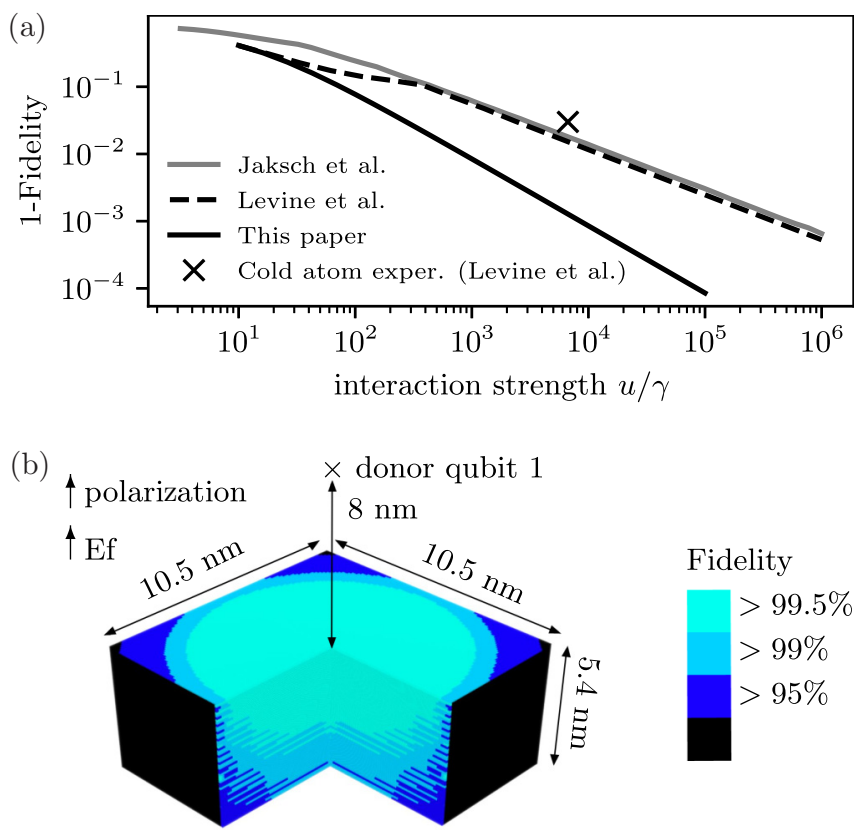

FIG. 6. Predicted Bell-state creation fidelities for general Rydberg gates (a) and for $\mathrm{Si}: \mathrm{P}$ or $\mathrm{Si}: \mathrm{As}$ (b). (a) Results from the Lindblad simulation of the pulse protocols from Jaksch et al. [15] and Levine et al. [16], along with the protocol proposed in this paper and the experimental data on alkali atoms in optical tweezers [16,31]. The Rabi frequency is set to the optimal value found for each $u$. In particular, they are approximately one order of magnitude larger than the ones for the Jaksch et al. [15] and Levine et al. [16] gates. (b) Fidelity map (plotted for $95 \%$ and above) as a function of distance between donors shows that fidelities are insensitive to donor placement. Experimental lifetime $T 1=235$ ps, as shown in Table I. $|r\rangle$ is the $2 p 0$ state polarized along $\{0,0,1\}$. The same fidelity region appears, diametrically opposed with regard to donor $1.8-\mathrm{nm}$ minimum separation for avoiding the molecular limit in the $2 p 0$ state [32]. The fidelity is robust to donors belonging to different silicon sublattices, diffusing out of plane (Si:As diffusion $<1 \mathrm{~nm} \mathrm{[10])} \mathrm{or}$ inhomogeneous broadening.

isotopes. For the Si:P $1 s-2 p+-$ transition, this effect can result in a variation of excited-state energies by about $0.1 \mathrm{meV}$ as measured in Ref. [23]. In our gate scheme, not taking into account this effect leads to an error in the detuning chosen for the pulse. In order to check that this leads to insubstantial changes in the gate fidelity, we plot the infidelity for a large range of detunings around the optimal value in Fig. 5 for a fixed Rabi frequency $\Omega=1000 \gamma$ (corresponding to $2.8 \mathrm{meV}$ for the Si:P lifetime, such that a variation of $0.1 \mathrm{meV}$ in the detuning corresponds to about $0.04 \Omega$ ). The fidelities are not heavily affected, and we conclude that the gate does not need tuning to account for inhomogeneous broadening.

\section{CONCLUSION}

To conclude, we have proposed a Rydberg gate, adapted to situations where Rabi frequency and interactions are of the same magnitude. While this is challenging in cold atoms, we have shown that they can be implemented in donors in silicon, reaching fidelities of $99.9 \%$ and having a high tolerance for donor placement error, opening the possibility to implement entangling gates in solid-state quantum computers with ion implantation [33]. The insensitivity to variations in the interaction strength and the inhomogeneous broadening not only brings advantages for scalability, but also reduces fine tuning in device operation and may therefore be advantageous for other solid-state platforms. We emphasize that the physical nature of the interactions is irrelevant, in particular, both exchange and dipolar interactions may be used. Acceptors do not have multivalley oscillations [34] and would therefore be ideal candidates for this robust gate implementation, as their dipolar interactions may be large enough to obtain high fidelities over extremely large areas, leading to a robust, long-range entangling gate in the solid state.

\section{ACKNOWLEDGMENTS}

We thankfully acknowledge discussions with R. Crane, S. Ebadi, N. J. Curson, W. Wu, E. Orlova, H. Pichler, and B. N. Murdin. We gratefully acknowledge financial support from the UK Engineering and Physical Sciences Research Council (ADDRFSS, Grant No. EP/M009564/1). A.S. acknowledges financial support from the International Max Planck Research School for Quantum Science and Technology (IMPRS-QST) funded by the Max-Planck-Gesellschaft (MPG).

\section{APPENDIX A: RYDBERG GATES IN THE PRESENCE OF DECOHERENCE}

First, we present the original resonant gate and its optimization [15]. Second, we present the recently proposed ultrafast off-resonant blockade gate [16]. The gate we propose and discuss in the main text shows much higher fidelities than the two previous proposals.

\section{Resonant blockade gate [15]}

The resonant Rydberg blockade gate requires the qubits to be individually addressable and the laser to be on resonance with the orbital transition. The pulse sequence (cf. Fig. 7) applies a $\pi$ pulse to the first atom, a $2 \pi$ pulse to the second atom, and a $\pi$ pulse to the first atom again. In the initial state $|00\rangle$ the pulse sequence has no effect on the qubits because the $|0\rangle$ state is off resonance with the laser. Due to the Rydberg blockade, $|11\rangle$ acquires the same phase as $|10\rangle$, as the second atom cannot be excited to $|r\rangle$, in total implementing the truth table of a controlled- $Z$ gate.

In the presence of a nonzero decoherence rate $\gamma$, there is an optimum for the Rabi frequency $\Omega$ : if $\Omega \approx \gamma$, the fidelity is low because the pulses take too long; however, if $\Omega \approx u$, the fidelity is also low as the blockade condition is not well fulfilled. We hence optimize the Rabi frequency $\Omega$ to maximize the fidelity $\mathcal{F}$.

In Fig. 7 we show that this intuitive picture is correct, i.e., there is a clear optimum value for the Rabi frequency. Interestingly, the optimal Rabi frequencies are rather large, showing that the gate operation time should be as small as possible to reduce loss from the excited state. This means that the hierarchy of scales is given by $\gamma \ll \Omega<u$. Importantly for the purposes of donor implementation, we also find that for a given value of the Rabi frequency, the fidelity is only weakly 


$$
\begin{array}{c|cccc}
\text { Pulse sequence } & |11\rangle & |10\rangle & |01\rangle & |00\rangle \\
\hline \text { 1. } \pi \text {-pulse on atom 1 } & \mathrm{i}|r 1\rangle & \mathrm{i}|r 0\rangle & |01\rangle & |00\rangle \\
\text { 2. } 2 \pi \text {-pulse on atom 2 } & \mathrm{i}|r 1\rangle & \mathrm{i}|r 0\rangle & \mathrm{i}|0 r\rangle & |00\rangle \\
\text { 3. } \pi \text {-pulse on atom 1 } & -|11\rangle & -|10\rangle & -|01\rangle & |00\rangle
\end{array}
$$

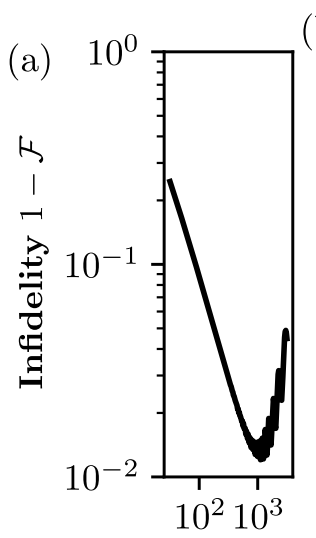

(b)

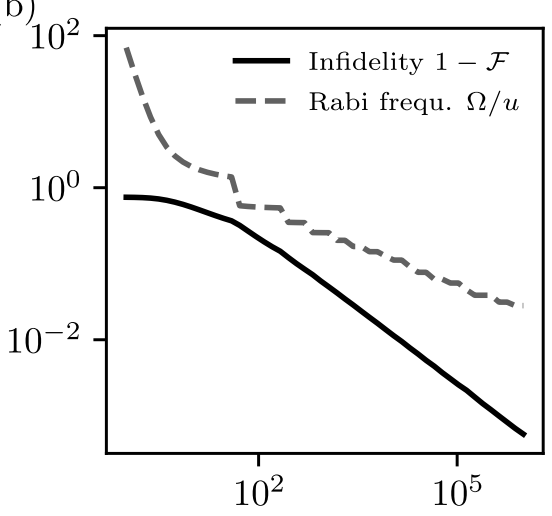

Rabi. frequ $\Omega / \gamma$ Interaction strength $u / \gamma_{\mathrm{se}}$

FIG. 7. Fidelity of the resonant Rydberg blockade gate. Table: Summary of the pulse sequence [15] in the ideal case of $u / \Omega=\infty$ and $u / \gamma=\infty$, showing the implementation of the controlled- $Z$ truth table. (a) Optimal Bell-state $\left|\Phi^{+}\right\rangle$fidelity obtained from a Lindblad simulation of the pulses, as a function of Rabi frequency for a fixed interaction strength $u=10^{4} \gamma_{\mathrm{se}}$ : there is a clear optimal value. (b) Optimal fidelities as a function of interaction strength, showing that modest fidelities can be already reached for small values of the interaction strength.

dependent on the exact value of $u$. For large $u$, a plateau is reached beyond which the fidelity does not increase due to decoherence processes then being the limiting factor. At this point, the Rabi frequency should be increased to increase fidelities further.

\section{Off-resonant blockade gate [16]}

In the improved blockade gate as presented and implemented in Ref. [16], only two global pulses with fixed detuning $\Delta \neq 0$ are needed. The pulse sequence proceeds as follows:

(1) Evolve for time $\tau$ with $\Omega_{1}=\Omega_{2}=\Omega$,

(2) Evolve for time $\tau$ with $\Omega_{1}=\Omega_{2}=\Omega \exp (i \xi)$,

followed by a single-qubit phase gate on both qubits [cf. Fig. 8(a)]. In the above sequence, the gate time $\tau$ is chosen such that the state $|11\rangle$ returns to itself after the first pulse, i.e., to the time corresponding to a blockaded $2 \pi$ pulse, $\tau=$ $2 \pi / \sqrt{2 \Omega^{2}+\Delta^{2}}$. Similarly, the phase $\xi$ of the laser in the second pulse is chosen such that the state $|01\rangle$ returns to itself after both pulses. Finally, the detuning $\Delta$ is chosen such that the phase acquired by both states differs by $\pi$ [in the sense that this phase difference remains after application of the global single-qubit phase gate, cf. Fig. 8(b)].

In the strongly interacting regime, the parameters were analytically calculated to be given by [16]

$$
\begin{gathered}
\Delta / \Omega=0.377371, \\
\xi=3.90242 .
\end{gathered}
$$

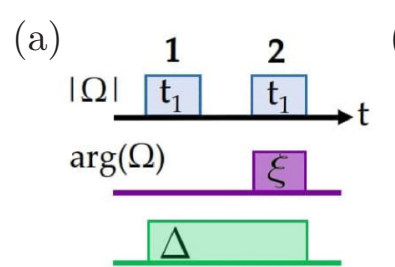

(b) $|00\rangle \rightarrow|00\rangle$

$$
\begin{aligned}
& |01\rangle \rightarrow|01\rangle e^{i \phi} \\
& |10\rangle \rightarrow|10\rangle e^{i \phi} \\
& |11\rangle \rightarrow|11\rangle e^{i(2 \phi-\pi)}
\end{aligned}
$$

(c)

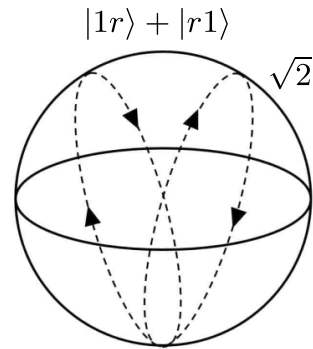

$|11\rangle$

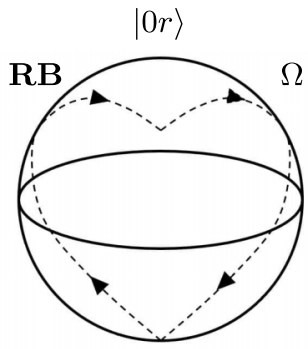

$|01\rangle$

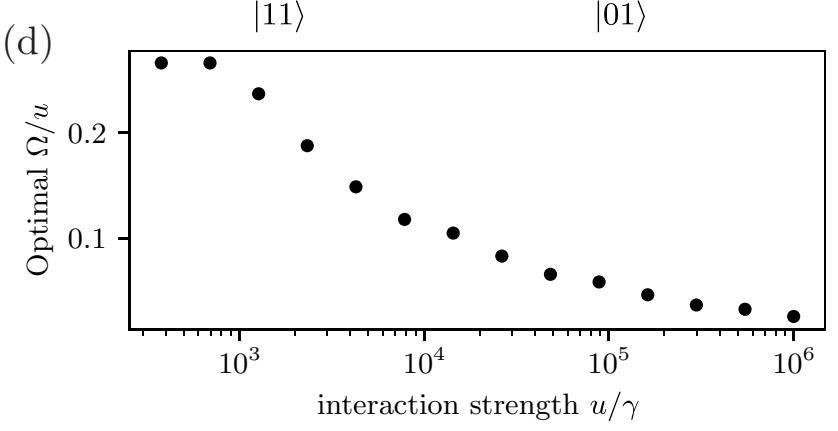

FIG. 8. Pulse sequence of the off-resonant gate adapted from Ref. [16]. (a) Global pulse sequence acting with $|\Omega|$ on both qubits simultaneously: $t_{1}$ pulse duration chosen such that the first pulse returns $|11\rangle$ to itself (whereas $|01\rangle$ and $|10\rangle$ are left in an arbitrary location on the Bloch sphere), $\xi$ phase of the second pulse (evolve with $\Omega \exp (i \xi))$ chosen such that $|01\rangle$ and $|10\rangle$ return to themselves after the pulse sequence, $\Delta$ detuning of the $|1\rangle$ to $|r\rangle$ transition chosen such that the phases highlighted in (b) are equal. Finally, the third step is to apply single-qubit phase gates to both qubits, with phase $\phi$, which corrects for global single-qubit phases built up in the dynamics. (b) Mapping of the qubit states due to the $\mathrm{Cz}$ gate. (c) The dynamics of the states $|11\rangle$ (with Rabi frequency $\sqrt{2} \Omega$ in the case of a perfect blockade) and $|01\rangle$ (with Rabi frequency $\Omega$, leading to a different path over the Bloch sphere) in terms of two-level systems. (d) Optimal Rabi frequency in the off-resonant blockade gate as a function of interaction strength and decoherence time.

This leaves the Rabi frequency as a free parameter, which we optimize for a given interaction strength $u$ and decoherence rates $\gamma_{\mathrm{se}}$ and $\gamma_{\mathrm{de}}=0.5 \gamma_{\mathrm{se}}$. Similarly to the resonant gate, we find a clear optimal Rabi frequency for a given interaction strength, which we show in Fig. 8 and find slightly higher fidelities than for the resonant gate (see main text).

A major limitation for both blockade gate proposals is the hierarchy of scales $\gamma \ll \Omega<u$, given by the requirement for the blockade condition to be fulfilled. This limits the maximum Rabi frequency, setting a lower bound for the gate duration and leaving more time for decoherence processes to kick in. Our calculation implies that the experiment in Ref. [16] operated very close to the optimal Rabi frequency, in part explaining their improvements over previous results. 


\section{APPENDIX B: FINITE-ELEMENT METHOD MULTIVALLEY WAVE FUNCTIONS}

In the following we explain how to obtain multivalley wave functions of donor electrons in silicon, which result from coupling the hydrogenic wave functions (envelope functions) into the manifolds determined by the symmetry group of the system. The envelope wave functions can be obtained by solving the Schrödinger equation for hydrogen, with the appropriate effective mass. We do this using the finite-element method [35] (developed in engineering for numerically computing approximations to solutions to partial differential equations and recently applied to donors [35]).

In the effective mass approximation states that close to a band extrema, such as the conduction-band minimum, each electron can be described by a mean-field Hamiltonian (every electron experiences the same average periodic potential), which is that of a single free electron with a modified mass (the effective mass) in an impurity potential.

Silicon has a face-centered cubic lattice which leads to its Brillouin zone (its primitive cell in reciprocal space) being a truncated octahedron. The latter contains six square faces, the centers of which are called $\mathrm{X}$ points. The conduction-band minima in silicon are positioned at the $X$ points and are all equivalent. Using the $\mathbf{k} \cdot \mathbf{p}$ method, the effective mass at a band extrema, such as the conduction-band minima, can be determined. The effective mass corresponds to the dispersion of the energy $E_{k}$ as a function of momentum $k\left(E_{k}=\frac{\hbar^{2} k^{2}}{2 m}\right)$. In silicon, the conduction-band minima are anisotropic: they are ellipsoids which lie along the axis linking the center of the Brillouin zone ( $\Gamma$ point) to the valley's $X$ point. In valleyspecific coordinates, we set this to be the $z$ axis. The effective mass along $z$ is referred to as longitudinal $m_{l}^{*}=0.191 m_{e}$, whereas along $x$ and $y$ it is transverse $m_{t}^{*}=0.916 m_{e}$, in reference to the conduction-band minimum ellipsoid, where $m$ is the mass of a free electron. The kinetic energy term in valley-specific coordinates is then

$$
-\frac{\hbar^{2}}{2}\left(\frac{\partial^{2}}{\partial x^{2}}+\frac{\partial^{2}}{\partial y^{2}}+\gamma \frac{\partial^{2}}{\partial z^{2}}\right)
$$

where $\gamma=m_{t} / m_{l}$.

The impurity potential is the Coulomb potential of the proton, which at large distances can be approximated as a point charge at the nucleus, felt by the valence electron:

$$
V_{\mathrm{imp}}(r)=-\frac{\hbar}{4 \pi \epsilon_{0} \epsilon_{r}} \frac{e^{2}}{r},
$$

where $e$ is the electron charge. However, the valley degree of freedom in multivalley semiconductors allows the wave functions to have a non-negligible probability of being at the core where the Coulomb approximation breaks down. To account for this, we add another potential to the Hamiltonian, widely referred to as the central-cell potential $U_{c c}(r)$, which takes the form of a $\delta$ function at the core and which we model using a Heaviside step function [36]. The value of the central-cell potential is determined using a bisection algorithm by requiring the energy of the solution of the FEM method to match the experimentally determined energies for the multivalley states.

It is also possible to add to the Hamiltonian the confining potential of an external electric field applied along the real- space $z$ axis (which corresponds to the $z$ axis in valley-specific coordinates), which is

$$
E_{f}(r)=e E_{f} z
$$

The Schrödinger equation, which we will simplify in the following, in the valley-specific coordinates where $z$ is the valley axis, is

$$
\left[-\frac{\hbar^{2}}{2 m *} \nabla^{2}+V_{\mathrm{imp}}(r)+U_{c c}(r)+E_{f}(r)\right] \psi_{i}(r)=\epsilon_{i} \psi_{i}(r) .
$$

The wave function is written

$$
\psi(r)=F_{i}(r) \phi\left(k_{j}, r\right) \alpha_{i} .
$$

It is composed of the envelope function $F_{i}(r)$, where the indices $i$ run over the hydrogenic states; the Bloch wave function $\phi\left(k_{j}, r\right)=e^{-i k_{j} \cdot r} u_{k_{j}}(r)$, which is the product of a plane wave and a lattice periodic function $u_{k_{j}}(r)$, where $j$ runs over the six valleys; and lastly, a multivalley parameter $\alpha_{i}$, which couples the function into various manifolds according to the $T_{d}$ point group mentioned above. We use the finite-element method to obtain $F_{i}(r)$ and couple it to the Bloch wave function and into the multivalley manifolds in a second step. As all the conduction-band minima are equivalent, the solution to this Schrödinger equation suffices for all the manifolds representing the $T_{d}$ point group.

We write the Hamiltonian in the atomic units $a_{B}=$ $4 \pi \epsilon_{0} \epsilon_{R} / e^{2} m_{t}$ and $E_{H}=e^{4} m_{t} /\left(\hbar 4 \pi \epsilon_{0} \epsilon_{R}\right)^{2}$ :

$$
\begin{aligned}
H= & \frac{-E_{H} a_{B}^{2}}{2}\left(\frac{\partial^{2}}{\partial x^{2}}+\frac{\partial^{2}}{\partial y^{2}}+\gamma \frac{\partial^{2}}{\partial z^{2}}\right)-\frac{E_{H} a_{B}}{\sqrt{x^{2}+y^{2}+z^{2}}} \\
& +U_{c c} \delta(x, y, z)+\frac{E_{h}}{a_{B} e} e E f z .
\end{aligned}
$$

We make four steps to simplify the Hamiltonian to improve FEM convergence:

(i) Transform to an anisotropic frame where $z^{\prime}=\sqrt{z}$ to have a symmetrized Hamiltonian.

(ii) Transform to spherical polar coordinates (the kinetic energy has spherical symmetry so this is allowed).

(iii) Choose the wave function $F_{i, m}(r)=e^{i m \phi} f_{j, m, \mu}(r, \theta)=$ $e^{i m \phi} \frac{1}{r} Y_{j, m, \mu}(r, \theta)$, where $m$ is the magnetic quantum number, to obtain a $2 \mathrm{D}$ Hamiltonian.

(iv) Transform the semi-infinite plane to a finite rectangle (tangent space) $r=r_{0} \tan \eta$ with $\eta \in\left[0, \frac{\pi}{2}\right]$ and $r_{0}$ a scaling factor which is best chosen comparable to the wave function of interest [35] for a compressed Hamiltonian. $Y_{j, m, \mu}(r, \theta)$ becomes $y_{j, m, \mu}(\eta, \theta)$.

The boundary conditions are then that the wave function must decay at infinity, which corresponds to $\alpha \rightarrow 0$ and $\alpha \rightarrow \frac{\pi}{2}$ :

$$
y(0, \theta)=y(\pi / 2, \theta)=0 .
$$

Additionally, for wave functions with parity and magnetic quantum numbers of opposite parity $y(\eta, \theta)=-y(\eta, \pi-\theta)$; 


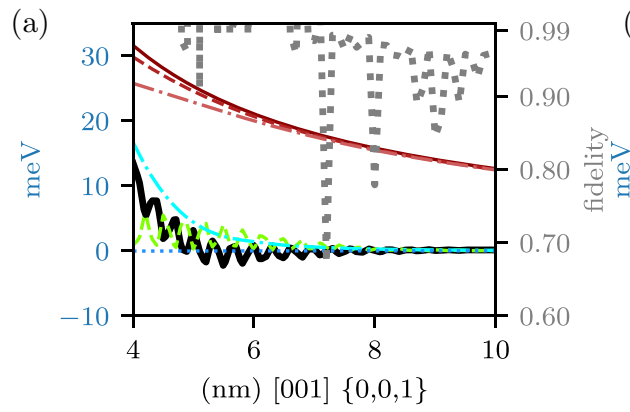

(d)

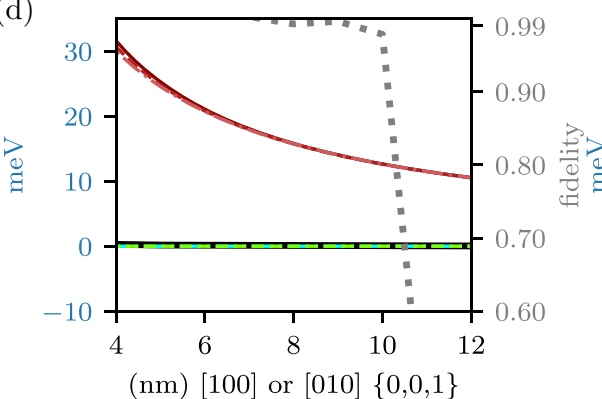

(b)

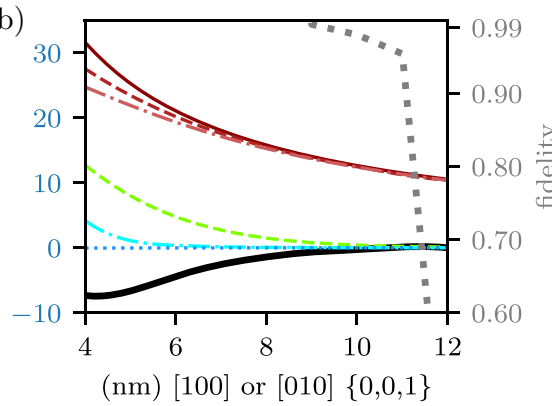

(e)

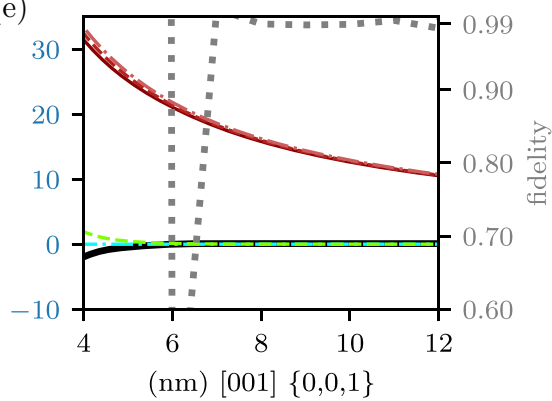

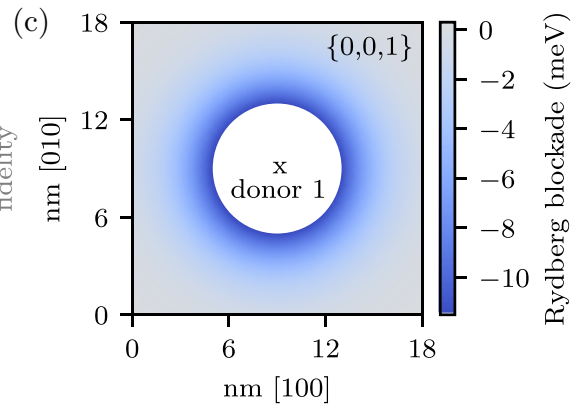

FIG. 9. Rydberg blockade between ${ }^{77} \mathrm{Se}+$ excited states with polarization $\{0,0,1\}$. Row 1 : interactions between two $2 p 0$ states and fidelity assuming a lifetime of $1 \mathrm{~ns}$. Row 2: interactions between two $1 s T_{2} \Gamma_{7}$ states and fidelity for the experimentally determined lifetime $7.7 \mathrm{~ns}$ [5]. Color labeling identical to Fig. 2 in main text. Reds $W$ : intersite Coulomb electron-electron repulsion ( $r r: 2 p 0-2 p 0, r g: 2 p 0-1 s A$, and $g g$ : $1 s A-1 s A)$. Green, $J$ : ferromagnetic exchange ( $2 p 0-2 p 0)$. Light blue, $V_{V d W}$ : van der Waals $(2 p 0-2 p 0)$. Dark blue, $V_{V d d}$ : induced dipole interaction with nonionizing field (negligible in all cases shown above), $u$ : total interaction calculated from Eq. (12). (a, d) Interdonor axis parallel to polarization axis. (b, e) Interdonor axis perpendicular to polarization axis. (c, f) Map of total interaction (meV) for one donor placed at the center of the map and the other to occupy any other position. White: avoiding the molecular limit. (a) Oscillation due to $J$. (e) Fidelity dips to 0 at $6 \mathrm{~nm}$ because $u$ changes sign, for which one can change the sign of the detuning. Fidelity remains large due to a difference on the order of $0.1 \mathrm{meV}$ coming from the $W$ terms and the extremely long decay time of the $1 s T_{2} \Gamma_{7}$ state.

therefore at the boundary

$$
y(\eta, \pi / 2)=-y(\eta, \pi / 2)=0 .
$$

The final symmetrized polar compressed 2D Hamiltonian reads

$$
\begin{aligned}
H= & -\frac{E_{H} a_{B}^{2}}{2 r_{0}}\left[\cos ^{2} \eta \frac{\partial}{\partial \eta}\left(\frac{m^{2} \cot ^{2} \eta}{\sin ^{2} \theta}\right)\right. \\
& \left.+\frac{\cot ^{2} \eta}{\sin \theta} \frac{\partial}{\partial \theta}\left(\sin \theta \frac{\partial}{\partial \theta}\right)\right]-\frac{E_{H} a_{B} r_{0}^{-1} \cot \eta}{\sqrt{1-(1-\gamma) \cos ^{2} \theta}} \\
& +\frac{U_{c c}}{\frac{4}{3} \pi r_{c}^{3}} H\left(r-r_{c}\right)+\sqrt{\gamma} r \cos \theta E_{f} U s\left(r-r_{m}\right),
\end{aligned}
$$

where $H(r)$ is the Heaviside $\Theta$ function, $U s(r)$ is the unit step function, and $r_{c}$ and $r_{m}$ the cutoff values. The solutions obtained via FEM are then numerically normalized using the Cuba Vegas package [37].

We now describe the coupling to the multivalley manifolds. Firstly, we reduce the six-valley problem $(-x, x,-y, y,-z, z)$ to an effective three-valley problem $(x, y, z)$ with $x, y$ and $z$ $\in[-\infty, \infty]$. We associate to each valley the envelope function in rotated Cartesian coordinates to ensure consistency when all the valleys will be coupled; considering we took $z$ as the valley axis we create a three-part vector corresponding to the envelope wave function in the three effective valleys: $\left[F_{i}(z, x, y), F_{i}(y, z, x), F_{i}(x, y, z)\right]$.
We transform the six functions of the tetrahedral point group into the effective three valleys by taking the Bloch wave function into account and using trigonometric identities to couple two valleys of opposite sign, each with a plane-wave factor $e^{-i k_{i} x}$ into one with $\sin 2 k_{i} x$ if the manifolds have an even total sign or $\cos 2 k_{i} x$ if it is odd. For the $1 s T_{2} \Gamma_{7}$ state, for example:

$$
\begin{aligned}
& F_{i}(x) e^{-i k_{i} x}(1,-1,0,0,0,0) \\
& \quad=F_{i}(x) e^{-i k_{i} x}-F_{i}(x) e^{-i k_{i} x} \\
& =2 i \sin 2 k_{i} x F_{i}(x) .
\end{aligned}
$$

We then normalize the wave functions.

\section{APPENDIX C: RYDBERG BLOCKADE RESULTS FOR Si:Se+ DONORS}

We study the interactions between two ionized selenium ( $\mathrm{Si}: \mathrm{Se}+$ ) donors in silicon to find out whether they might be good candidates for Rydberg gates. Si:Se+ donors have direct transitions in the infrared regime, and their optical characteristics been studied for decades and have recently undergone renewed interest in the context of providing a spin-photon interface for the silicon platform [17].

As can be seen in Fig. 9, oscillations in the exchange energy can be seen when the donors are aligned along the polarization axis [Figs. 9(a) and 9(d)] but not when perfectly aligned along the axis perpendicular to the polarization 
[Figs. 9(b) and 9(e)]. In the case of the $1 s T 2$ state, the exchange is so small that the oscillations can hardly be seen in Fig. 9(d). Figures 9(c) and 9(d) are top views of the Rydberg interactions [also represented in Figs. 9(b) and 9(e)], of direct use when calculating the fidelity over large physical areas. For the $1 s T 2$ state, the Rydberg interaction changes sign due to the rapid decay of the exchange, which causes a dip in the fidelity.

In Figs. 9(a), 9(b) 9(d), and 9(e), the fidelity of the entangling gate proposed in the main text is also plotted. The oscillation in the Rydberg interaction caused by the exchange is so steep that the fidelity has sharp oscillations which are not very well resolved, despite the $0.1-\mathrm{nm}$ step size of the calculation. For the $1 s T 2 \gamma 7$ state, however, the long $|r\rangle$ lifetime of $7.7 \mathrm{~ns}$ puts the fidelity extremely high, despite the small magnitude of $u$.

\section{APPENDIX D: ELECTRIC FIELDS}

\section{Ionization rates}

The ionization rate for a hydrogen atom in an excited state is given in [28] to be

$$
\frac{1}{\tau}=n-3\left[n_{2} !\left(n_{2}+|m|\right) !\right]^{-1}\left(n^{3} \frac{E_{f}}{4}\right)^{-2 n_{2}-|m|_{-1}} e^{3\left(n_{1}-n_{2}\right) \frac{-2}{3 n^{3} E_{f}}},
$$

with $n, m, n_{1}$, and $n_{2}$ are the principal, magnetic, and parabolic quantum numbers, respectively (in this paper we consider the $2 p 0$ state, which has $\left.n_{1}=1, n_{2}=0\right)$, and $E_{f}$ is the applied field strength in atomic units. We scale the results for the hydrogen atom by using experimental results acquired by [38] on $\mathrm{Si}: \mathrm{P}$ in the ground state to determine the atomic units for the electric field used in Eq. (D1). For an atom in the ground state Eq. (D1) reduces to

$$
\frac{1}{\tau}=\frac{\omega \alpha}{F} \exp \left[-\frac{\alpha}{F}\right]
$$

originally given in [39], with $\alpha=4 \sqrt{2 m_{t}} E_{f}^{3 / 2} /(3 e \hbar)$ the atomic field and $\omega=12 E_{b} / \hbar$, where $E_{b}$ is the binding energy, $e$ is the electron mass, and $m_{t}$ is the tunneling mass. The tunneling mass depends on the direction in which the electric field is applied, with the [111] direction yielding the slowest tunneling times [38]. The 2D Hamiltonian which enables us to use the FEM for investigating induced dipolar interactions in this paper requires us to consider applying the electric field along [100]. The minimum ionization rate is given by the Bohr period $\tau_{B}=2 \pi \hbar / E_{b}$ [38], on the order of $10^{-13} \mathrm{~s}$ for Si:P and $10^{-14} \mathrm{~s}$ for $\mathrm{Si}: \mathrm{Se}+$.

The results of these formula comply with experimental results for the $\mathrm{Si}: \mathrm{P} 1 s A$ state [38]. The classical ionization threshold for $\mathrm{Si}: \mathrm{P} 2 p 0$ is at $0.28 \mathrm{~V} / \mu \mathrm{m}$. In order to reduce the ionization probability during the $2 p 0$ lifetime of $235 \mathrm{ps,}$ we can see from Fig. 10 that the electric field should be on the order of $0.2 \mathrm{~V} / \mu \mathrm{m}$.

The results for the theoretically calculated ionization rates for $\mathrm{Si}: \mathrm{Se}+$ can be found in Fig. 10. The $2 p 0$ result was obtained by inserting the $1 s A \alpha$ and $\omega$ values into Eq. (D1). The result for $1 s T_{2}\left(n_{1}=0, n_{2}=0\right)$ coincides with the result obtained when calculating $\alpha$ and $\omega$ values from the binding energy of $1 s T_{2}$ and directly using Eq. (D2). In order to reduce
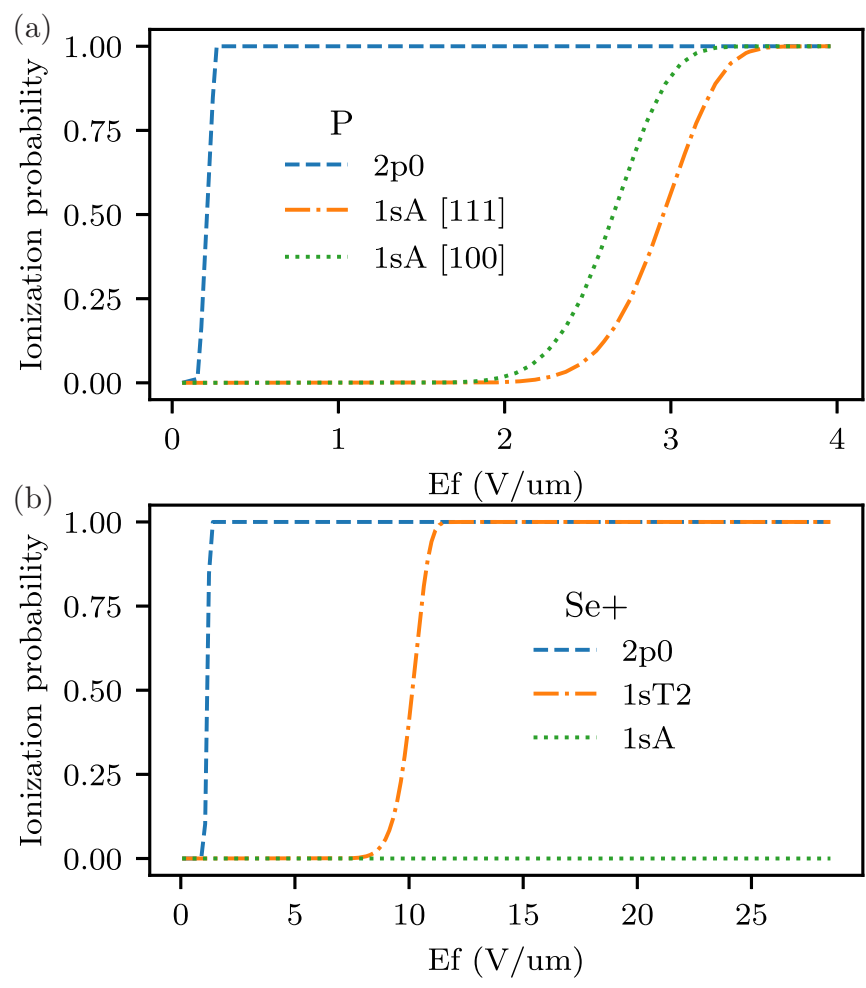

FIG. 10. Ionization probability within experimentally determined lifetimes. (a) Ionization probability within $200 \mathrm{ps}$ for various $\mathrm{Si}: \mathrm{P}$ states and electric field directions. Checked against experimental data from [38]. (b) Ionization probability within $7.7 \mathrm{~ns}$ for theoretical values for $\mathrm{Si}: \mathrm{Se}+2 p 0$ (blue), $1 s T_{2} \Gamma_{7}$ (orange), $1 s A$ (green). Electric field applied along [100].

the ionization probability during the $1 s T_{2}$ experimentally measured lifetime of $7.7 \mathrm{~ns}$, we can see from Fig. 10 that the electric field should be on the order of $8 \mathrm{~V} / \mu \mathrm{m}$ for $1 s T_{2}$ and 1 $\mathrm{V} / \mu \mathrm{m}$ for $2 p 0$. In effect, these electric fields yield negligible dipolar forces, as can be seen from Fig. 3 .

\section{Stark shifts}

In this paper we propose a simple way to calculate the exact single-valley Stark shift of donors using the FEM by including a slope corresponding to the electric field in the Schrödinger equation in Eq. (B4). This single-valley Stark shift enables us to check our multivalley, perturbatively calculated Stark shift because it includes contributions from the continuum. This is only possible for a field applied along the polarization axis as, when applied perpendicular the Schrödinger equation, it no longer reduces to a 2D Hamiltonian.

Multivalley oscillations are on the same scale as the singlevalley wave function for the singly ionized donor $\mathrm{Se}+$, in which the valence electron is tightly pulled in towards the core. All Stark shifts are plotted in Fig. 11 and are very close in magnitude for both methods, single valley or multivalley, for electric fields smaller than the ionizing electric fields for the donor-excited-state lifetimes previously determined.

The perturbatively calculated single-valley calculations include single $s$ states with no central-cell corrections (ccc) in the $2 p 0$ calculations and with a ccc for the $1 s T_{2} \Gamma_{7}$ calculation 

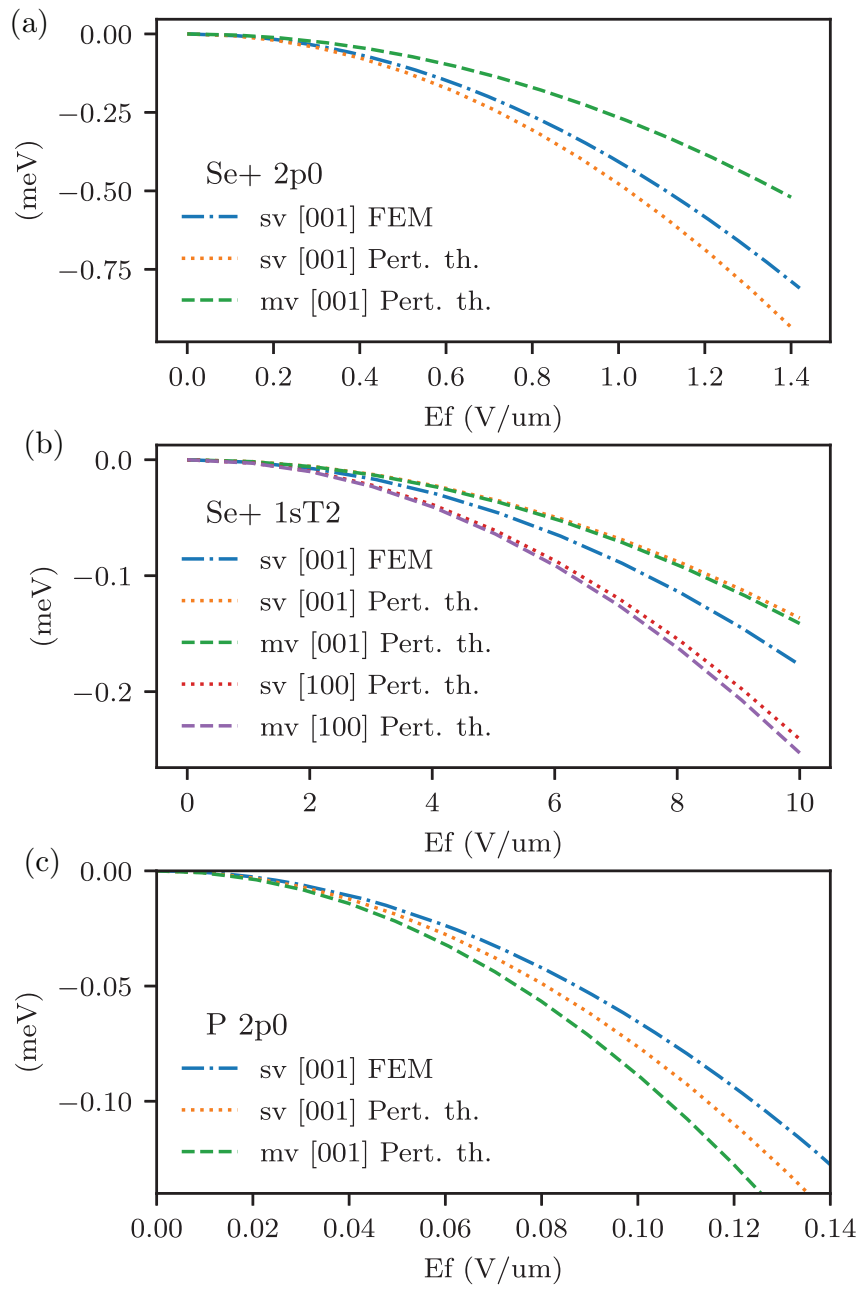

FIG. 11. Stark shifts for $E_{f}$ smaller than the ionization limit determined in Fig. 10. The FEM result includes contributions from the conduction-band states not included in the perturbative results. (a) $\mathrm{Si}: \mathrm{Se}+2 p 0$ Stark shift. (c) $\mathrm{Si}: \mathrm{Se}+1 s T_{2} \Gamma_{7}$ Stark shift. (b) Si:P $2 p 0$ Stark shift.

in order to be able to compare directly to the FEM results. We can see both results are close, despite the perturbative results not including contributions from the continuum. The multivalley calculations include, for the ground as well as for higher-lying $s$ states, A, E, and T manifolds, each with their own ccc.

For the $2 p 0$ states of both donors, the main contributing state is $2 s$ in the single-valley case, and $2 s E$ and $2 s A$ in the multivalley case. For Se+ $1 s T_{2} \Gamma_{7}$ in both single and multivalley cases, the main contributions to the Stark shift parallel to the polarization of the state come from the $p 0$ states and perpendicular to the polarization comes from the $p+$ states.

Considering these Stark shifts, with laser linewidths below $0.1 \mathrm{meV}$ and Rabi frequencies on the order of $1 \mathrm{meV}$, small electric fields would suffice to shift the donors on and off resonance. Large fields could even be applied to all the ground-state donors not contributing to the gate to fully ensure they are of-resonance with the laser exciting to the Rydberg state, because ground-state donors can resist far larger fields than the excited states, which have a short lifetime and higher ionization probabilities.

\section{Stray electric fields}

In this section we show that the stray electric fields from the gates local to the surrounding donors (Stark shifting them off resonance with the laser) do not interfere with the gate operation. In order to reduce the probability of the laser exciting a transition, the detuning must be increased proportional to the Rabi frequency, for example, for a 0.1 probability, $\Delta=4 \Omega$. For realistic Rabi frequencies between $10^{8}$ and $10^{11} \mathrm{~Hz}$, depending on the experimental laser power, this sets an upper bound on $\Delta$ of around $0.4 \mathrm{meV}$.

The $2 p 0$ state in Si:P ionizes in relatively small fields, as can be seen in the previous section entitled Ionization rates and Stark shifts. A $0.4-\mathrm{meV}$ Stark shift would require an electric field of $0.5 \mathrm{~V} / \mu \mathrm{m}$, which we calculated using the multivalley perturbation theory method described in that section.

As is shown in the figure in the main text, we use the finiteelement method to solve for the electric fields in the proposed geometry. We find that if the left local gates of donors not participating in the entangling operation are held at $-0.0025 \mathrm{~V}$ and the right ones at $0.0025 \mathrm{~V}$, with the gates surrounding the central donors all held at $0 \mathrm{~V}$, and the top global gate at $0.015 \mathrm{~V}$ and the bottom one at $0.015 \mathrm{~V}$, then the donors not participating in the entangling operation will get shifted off resonance with the laser with a field of $0.5 \mathrm{~V} / \mu \mathrm{m}$, and the two donors participating in the readout, in the case of $\mathrm{Si}: \mathrm{P}$ $2 p 0$ being chosen as $|r\rangle$, will get a small field parallel to the interdonor axis due to the global gates, of around $0.18 \mathrm{~V} / \mu \mathrm{m}$, which will not ionize them within the excited-state lifetime but will induce dipolar interactions. We conclude that with this proposed geometry, there are no unwanted stray electric fields.
[1] J. T. Muhonen, A. Laucht, S. Simmons, J. P. Dehollain, R. Kalra, F. E. Hudson, S. Freer, K. M. Itoh, D. N. Jamieson, J. C. McCallum, A. S. Dzurak, and A. Morello, Quantifying the quantum gate fidelity of single-atom spin qubits in silicon by randomized benchmarking, J. Phys.: Condens. Matter 27, 154205 (2015).

[2] A. M. Tyryshkin, S. Tojo, J. J.L. Morton, H. Riemann, N. V. Abrosimov, P. Becker, H. J. Pohl, T. Schenkel, M. L. W.
Thewalt, K. M. Itoh, and S. A. Lyon, Electron spin coherence exceeding seconds in high-purity silicon, Nat. Mater. 11, 143 (2012).

[3] K. J. Morse, P. Dluhy, J. Huber, J. Z. Salvail, K. Saeedi, H. Riemann, N. V. Abrosimov, P. Becker, H.-J. Pohl, S. Simmons, and M. L. W. Thewalt, Zero-field optical magnetic resonance study of phosphorus donors in 28-silicon, Phys. Rev. B 97, 115205 (2018). 
[4] R. Lo Nardo, G. Wolfowicz, S. Simmons, A. M. Tyryshkin, H. Riemann, N. V. Abrosimov, P. Becker, H.-J. Pohl, M. Steger, S. A. Lyon, M. L. W. Thewalt, and J. J. L. Morton, Spin relaxation and donor-acceptor recombination of $\mathrm{se}^{+}$in 28-silicon, Phys. Rev. B 92, 165201 (2015).

[5] K. J. Morse, R. J. S. Abraham, A. DeAbreu, C. Bowness, T. S. Richards, H. Riemann, N. V. Abrosimov, P. Becker, H.-J. Pohl, M. L. W. Thewalt, and S. Simmons, A photonic platform for donor spin qubits in silicon, Sci. Adv. 3, e1700930 (2017).

[6] Y. He, S. K. Gorman, D. Keith, L. Kranz, J. G. Keizer, and M. Y. Simmons, A two-qubit gate between phosphorus donor electrons in silicon, Nature (London) 571, 371 (2019).

[7] F. Borjans, X. G. Croot, X. Mi, M. J. Gullans, and J. R. Petta, Resonant microwave-mediated interactions between distant electron spins, Nature (London) 577, 195 (2020).

[8] B. Koiller, X. Hu, and S. Das Sarma, Exchange in Silicon-Based Quantum Computer Architecture, Phys. Rev. Lett. 88, 027903 (2001).

[9] M. Fuechsle, J. a Miwa, S. Mahapatra, H. Ryu, S. Lee, O. Warschkow, L. C. L. Hollenberg, G. Klimeck, and M. Y. Simmons, A single-atom transistor, Nat. Nanotechnol. 7, 242 (2012).

[10] T. J. Z. Stock, O. Warschkow, P. C. Constantinou, J. Li, S. Fearn, E. Crane, E. V. S. Hofmann, A. Kölker, D. R. Mckenzie, S. R. Schofield, and N. J. Curson, Atomic-scale patterning of arsenic in silicon by scanning tunneling microscopy, ACS Nano 14, 3316 (2020).

[11] C. D. Hill, E. Peretz, S. J. Hile, M. G. House, M. Fuechsle, S. Rogge, M. Y. Simmons, and L. C. L. Hollenberg, Quantum Computing: A surface code quantum computer in silicon, Sci. Adv. 1, e1500707 (2015).

[12] P. T. Greenland, S. A. Lynch, A. F. G. Van Der Meer, B. N. Murdin, C. R. Pidgeon, B. Redlich, N. Q. Vinh, and G. Aeppli, Coherent control of Rydberg states in silicon, Nature (London) 465, 1057 (2010).

[13] R. de Sousa, J. D. Delgado, and S. Das Sarma, Silicon quantum computation based on magnetic dipolar coupling, Phys. Rev. A 70, 052304 (2004).

[14] M. D. Lukin, M. Fleischhauer, R. Cote, L. M. Duan, D. Jaksch, J. I. Cirac, and P. Zoller, Dipole Blockade and Quantum Information Processing in Mesoscopic Atomic Ensembles, Phys. Rev. Lett. 87, 037901 (2001).

[15] D. Jaksch, J. I. Cirac, P. Zoller, S. L. Rolston, R. Côté, and M. D. Lukin, Fast Quantum Gates for Neutral Atoms, Phys. Rev. Lett. 85, 2208 (2000).

[16] H. Levine, A. Keesling, G. Semeghini, A. Omran, T. T. Wang, S. Ebadi, H. Bernien, M. Greiner, V. Vuletić, H. Pichler, and M. D. Lukin, Parallel Implementation of High-Fidelity Multiqubit Gates with Neutral Atoms, Phys. Rev. Lett. 123, 170503 (2019).

[17] A. DeAbreu, C. Bowness, R. J. S. Abraham, A. Medvedova, K. J. Morse, H. Riemann, N. V. Abrosimov, P. Becker, H.-J. Pohl, M. L. W. Thewalt, and S. Simmons, Characterization of the Si:se ${ }^{+}$Spin-Photon Interface, Phys. Rev. Appl. 11, 044036 (2019).

[18] P. Clauws, J. Broeckx, E. Rotsaert, and J. Vennik, Oscillator strengths of shallow impurity spectra in germanium and silicon, Phys. Rev. B 38, 12377 (1988).

[19] M. J. Gullans and J. M. Taylor, Optical control of donor spin qubits in silicon, Phys. Rev. B 92, 195411 (2015).
[20] V. Tyuterev, J. Sjakste, and N. Vast, Theoretical intrinsic lifetime limit of shallow donor states in silicon, Phys. Rev. B 81, 245212 (2010).

[21] W. Royle, Modern quantum optics of mid gap chalcogen donors in single crystal silicon, Ph.D. thesis, Cardiff University, 2018.

[22] H.-W. Hübers, S. G. Pavlov, S. A. Lynch, Th. Greenland, K. L. Litvinenko, B. Murdin, B. Redlich, A. F. G. van der Meer, H. Riemann, N. V. Abrosimov, P. Becker, H.-J. Pohl, R. Kh. Zhukavin, and V. N. Shastin, Isotope effect on the lifetime of the $2 p_{0}$ state in phosphorus-doped silicon, Phys. Rev. B 88, 035201 (2013).

[23] K. L. Litvinenko, E. T. Bowyer, P. T. Greenland, N. Stavrias, J. Li, R. Gwilliam, B. J. Villis, G. Matmon, M. L. Y. Pang, B. Redlich, a. F. G. van der Meer, C. R. Pidgeon, G. Aeppli, and B. N. Murdin, Coherent creation and destruction of orbital wavepackets in Si:P with electrical and optical read-out, Nat. Commun. 6, 6549 (2015).

[24] M. Pioro-Ladrière, Y. Tokura, T. Obata, T. Kubo, and S. Tarucha, Micromagnets for coherent control of spin-charge qubit in lateral quantum dots, Appl. Phys. Lett. 90, 024105 (2007).

[25] E. Crane, T. Crane, A. Schuckert, N. H. Le, K. Stockbridge, S. Chick, and A. J. Fisher, Optically controlled entangling gates in randomly doped silicon, Phys. Rev. B 100, 064201 (2019).

[26] W. Wu and A. J. Fisher, Exchange between deep donors in semiconductors: A quantum defect approach, Phys. Rev. B 77, 045201 (2008).

[27] W. Heitler and F. London, Interaction between neutral atoms and homopolar binding according to quantum mechanics, in Quantum Chemistry, edited by H. Hettema, World Scientific Series in 20th Century Chemistry Vol. 8 (World Scientific Publishers, Singapore, 2000), pp. 140-155.

[28] T. Yamabe, A. Tachibana, and H. J. Silverstone, Theory of the ionization of the hydrogen atom by an external electrostatic field, Phys. Rev. A 16, 877 (1977).

[29] T. F. Watson, S. G. J. Philips, E. Kawakami, D. R. Ward, P. Scarlino, M. Veldhorst, D. E. Savage, M. G. Lagally, M. Friesen, S. N. Coppersmith, M. A. Eriksson, and L. M. K. Vandersypen, A programmable two-qubit quantum processor in silicon, Nature (London) 555, 633 (2018).

[30] D. S. Weiss and M. Saffman, Quantum computing with neutral atoms, Phys. Today 70(7), 44 (2017).

[31] H. Bernien, S. Schwartz, A. Keesling, H. Levine, A. Omran, H. Pichler, S. Choi, A. S. Zibrov, M. Endres, M. Greiner, V. Vuletić, and M. D. Lukin, Probing many-body dynamics on a 51-atom quantum simulator, Nature (London) 551, 579 (2017).

[32] J. Li, N. H. Le, K. L. Litvinenko, S. K. Clowes, H. Engelkamp, S. G. Pavlov, H. W. Hubers, V. B. Shuman, L. D. Portsel, D. N. Lodygin, Y. A. Astrov, N. V. Abrosimov, C. R. Pidgeon, A. Fisher, Z. Zeng, Y. M. Niquet, and B. N. Murdin, Radii of Rydberg states of isolated silicon donors, Phys. Rev. B 98, 085423 (2018).

[33] J. F. Ziegler, M. D. Ziegler, and J. P. Biersack, SRIM - The stopping and range of ions in matter (2010), Nucl. Instrum. Methods Phys. Res., Sect. B 268, 1818 (2010), 19th International Conference on Ion Beam Analysis.

[34] J. Zhu, W. Wu, and A. J. Fisher, Linear combination of atomic orbitals model for deterministically placed acceptor arrays in silicon, Phys. Rev. B 101, 085303 (2020). 
[35] N. H. Le, G. V. Lanskii, G. Aeppli, and B. N. Murdin, Giant non-linear susceptibility of hydrogenic donors in silicon and germanium, Light Sci. Appl. 8, 64 (2019).

[36] T. H. Ning and C. T. Sah, Multivalley effective-mass approximation for donor states in silicon, I. Shallow-level group-V impurities, Phys. Rev. B 4, 3468 (1971).
[37] T. Hahn, Cuba-A library for multidimensional numerical integration, Comput. Phys. Commun. 168, 78 (2005).

[38] N. Zurauskiene and A. Dargys, Impurity-band tunnelling anisotropy in n-Si, Semicond. Sci. Technol. 5, 813 (1990).

[39] L. D. Landau and E. M. Lifshitz, Quantum Mechanics: NonRelativistic Theory, Course of Theoretical Physics (Elsevier Science, New York, 1981). 